\title{
Mathematical model for the infectiology of brucellosis with some control strategies
}

\author{
Nkuba Nyerere ${ }^{1,5}$, Livingstone S. Luboobi ${ }^{2}$, Saul C. Mpeshe $e^{3}$ and Gabriel M. Shirima ${ }^{4}$ \\ ${ }^{1}$ Department of Applied Mathematics and Comp. Sciences, Nelson Mandela African Ins. of Science and Tech., Arusha, Tanzania \\ ${ }^{2}$ Institute of Mathematical Sciences, Strathmore University, Nairobi, Kenya \\ ${ }^{3}$ Department of Mathematics, University of Iringa, P.O. Box 200, Iringa, Tanzania \\ ${ }^{4}$ Department of Global Health and Bio-Medical Sciences, Nelson Mandela African Ins. of Science and Tech., Arusha, Tanzania \\ ${ }^{5}$ Department of Mathematics, Informatics and Computational Sciences, Sokoine University of Agriculture, Tanzania
}

Received: 21 June 2019, Accepted: 8 October 2019

Published online: 25 December 2019.

\begin{abstract}
Brucellosis is a neglected zoonotic infection caused by gram-negative bacteria of genus brucella. In this paper, a deterministic mathematical model for the infectiology of brucellosis with vaccination of ruminants, culling of seropositive animals through slaughter, and proper environmental hygiene and sanitation is formulated and analyzed. A positive invariant region of the formulated model is established using the Box Invariance method, the effective reproduction number, $R_{e}$ of the model is computed using the standard next generation approach. We prove that the brucellosis free equilibrium exists, locally and globally asymptotically stable if $R_{e}<1$ while the endemic equilibrium point exists, locally and globally asymptotically stable if $R_{e}>1$. Sensitivity analysis of the effective reproductive number shows that, natural mortality rate of ruminants, recruitment rate, ruminant to ruminant transmission rate, vaccination rate, and disease induced culling rate are the most sensitive parameters and should be targeted in designing of the control strategies for the disease. Numerical simulation is done to show the variations of each subpopulation with respect to the control parameters.
\end{abstract}

Keywords: Brucellosis, mathematical model, infectiology, environmental hygiene.

\section{Introduction}

Brucellosis is a zoonotic infection caused by gram-negative bacteria of genus brucella (B. abortus primarily from cattle, B. melitensis from small ruminants, B. suis from swine, and B. canis from dogs) [14,34,52,57]. It is considered by the Food and Agriculture Organisation (FAO), the World Health Organisation (WHO) and World Organization for Animal Health (Office International des Epizooties (OIE)) as one of the most widespread zoonoses in the world alongside bovine tuberculosis and rabies [45]. The disease is an ancient one that was described more than 2000 years ago by the Romans [24] and has been known by various names, including Mediterranean fever, Malta fever, gastric remittent fever, bang's disease, crimean fever, gibraltar fever, rock fever, lazybones disease and undulant fever [55]. A British military medical officer David Bruce isolated brucella bacteria from an infected individual's blood for the first time in 1887 and hence the disease was named brucellosis to honor his contribution [54]. Furthermore, in 1905 Zamitt carried out an experiment on goats to investigate the origin of human brucellosis, and found that, human brucellosis originates from goats [2]. To date, eight species have been identified and named primarily for the source animal or features of infection. Of these, the following four have moderate-to-significant human pathogenicity: Brucella melitensis (highest pathogenicity), Brucella suis (high pathogenicity) named after the source animal (swine), Brucella abortus (moderate pathogenicity) named after the feature of infection, Brucella canis named after the source animal (moderate pathogenicity) $[37,38,56]$. 
In animals, brucellosis is transmitted when susceptible animals ingest contaminated materials like tissues or discharges from infected animals while in humans the bacteria is transmitted by ingestion of contaminated unpasteurized milk or other dairy products. Furthermore, direct contact with aborted fetuses, discharges and occupational accidents through needle injection during mass vaccination and during laboratory manipulation is another route of brucellosis transmission in the human population. In this view, farmers, laboratory personnels, abattoir workers and veterinarians are more susceptible to the disease. Infected animals exhibit clinical signs that are of economic significance to stakeholders and include reduced fertility, abortion, poor weight gain, lost draught power, and a substantial decline in milk production [21, 53]. Symptoms in humans include: continuous or intermittent fever, headache, weakness, profuse sweats, chills, joint pains, aches, weight loss as well as devastating complications in pregnant women. Neurological complications, endocarditis and testicular or bone abscess formation can also occur $[13,16]$. The infection can also affect the liver and spleen, it may last for days or months, and sometimes for a year or more if not treated. The clinical signs in human present diagnostic difficulties because the disease can be confused with typhoid fever, malaria, rheumatic fever, joint diseases and relapsing fever. Human brucellosis is debilitating and requires prolonged treatment with combination of antibiotics [27].

The global burden of human brucellosis remains enormous: The infection causes more than 500,000 cases per year worldwide. The annual number of reported cases in United States (now about 100) has dropped significantly because of aggressive animal vaccination programs and milk pasteurization. Most US cases are now due to the consumption of illegally imported unpasteurized dairy products from Mexico. Approximately $60 \%$ of human brucellosis cases in the United States now occur in California and Texas [43].

In Africa Brucellosis exists throughout sub-Saharan Africa, but the prevalence is unclear and poorly understood with varying reports from country to country, geographical regions as well as animal factors [50]. Most African countries are of poor socioeconomic status, with people living with and by their livestock, while health networks, surveillance and vaccination programs are virtually non-existent. In Tanzania, the first outbreak of brucellosis was reported in Arusha in 1927 [48]. Previous surveys in Tanzania have demonstrated the occurrence of the disease in cattle in various production systems, regions and zones with individual animal level seroprevalence varying from 1 to $30 \%$. There has been isolation of Brucella for more than 50 years ago and at that time B. abortus and B. melitensis were isolated from cattle and small ruminants respectively. In humans, the average prevalence varies from 1 to 5\% [49], a recent study by [8]shows that brucellosis incidence is moderate in northern Tanzania and suggests that the disease is endemic and an important human health problem in this area. Moreover, special cases had been reported in areas of northern, eastern, lake and western zones with seroprevalence varying from 0.7 to $20.5 \%$. [46].

Despite the WHO, FAO, OIE efforts and interventions are available, brucellosis continues to pose great economic threat by affecting livelihood and food security in both developed and developing countries; it is endemic in most of the developing world and causes devastating losses to the livestock industry especially small-scale livestock holders, thereby limiting economic growth and hindering access to international markets [21] from generation to generation. Thus, there is a need to assess the current control strategies and their cost-effectiveness if we are to control or eradicate the disease. So far few studies $[3,25,33,39,40,44,56]$, have been developed to analyze dynamics of and spread of brucellosis in a homogeneous/heterogeneous populations. However, none of these studies have considered the mathematical approach for the impact of vaccination of ruminants, culling of seropositive animals through slaughter, and proper environmental hygiene and sanitation in reducing or eradicating the disease in cattle, small ruminants and human populations using mathematical models. This paper is at hand to fill the gap. 


\section{Model formulation}

\subsection{Dynamics of brucellosis}

In this section, we formulate a deterministic mathematical model for the transmission dynamics of Brucellosis in domestic small ruminants, cattle and human populations. The model includes: direct transmission of brucellosis within the cattle population, within small ruminants (sheep and goats) and from both species to human and indirect transmission from the environment to livestock and humans. Cattle and small ruminants newborns are either vaccinated or remain susceptible. Based on the epidemiological status, the cattle population at any time $t$ is divided into vaccinated $V_{c}(t)$, susceptible $S_{c}(t)$, and infective $I_{c}(t)$ classes. Similarly, the small ruminant population at any time $t$ is divided into vaccinated $V_{s}(t)$, susceptible $S_{S}(t)$, and infectious $I_{S}(t)$ subpopulations while the total human population, $N_{h}(t)$ at any time $t$ is divided into susceptible, $S_{h}(t)$, infected, $I_{h}(t)$ and recovered, $R_{h}(t)$ individuals. Susceptible cattle become infected when they are in contact with infected cattle (direct transmission) at the rate of $\beta_{c}$ or through contact with infected raw blood, meat, placentas, aborted fetus, unpasteurized milk or other dairy products (indirect transmission) at the rate $\alpha_{c}$, and susceptible small ruminants become infected when they are in contact with infectious small ruminants at the rate of $\beta_{s}$ or through contact with their products at the rate $\alpha_{s}$ while the transmission to humans is expressed as additive contributions of transmission from infective cattle, small ruminants and their products. Appertaining to the fact that it is very difficult to determine the quantity of brucella in environment, we define the average number of brucella that is enough for a host to be infected with brucellosis as an infectious unit and let $B(t)$ to be the number of infectious units in the environment. During the incubation period, Brucellosis is hardly detected, but individuals at this period can infect the susceptible individuals at the same transmission rate as the infectious individual and discharge the same quantity of brucella into the environment per unit time. It is against this background, we assume that individuals in the incubation period and post incubation period are hosted in the same population compartment called infectious. The interaction within and between the four populations shows that, veterinary surgeons, laboratory assistants, and farmers are predominantly exposed to the pathogen (See Figure $1)$.

\subsection{Model assumptions}

In formulation of the model, the following assumptions are taken into consideration:

(i) There is no direct transmission between cattle and small ruminants.

(ii) Infected animals shed the brucellosis pathogen in the environment.

(iii) Livestock seropositivity is a life-long lasting.

(iv) Immunized individuals cannot be infected unless their vaccine efficacy wanes.

(v) There is constant natural mortality rate in each of the species.

(vi) The mixing in each population is homogeneous.

(vii) The birth rate for each population is greater than natural mortality rate.

The variables and parameters used in this model are respectively summarized in Table 1 and Table 2.

\subsection{Compartmental Flow Diagram for the Disease Dynamics}

The interactions between the human, cattle, small ruminants populations and the brucella in the environment are illustrated in Figure 1. 
Table 1: Model Variables

\begin{tabular}{ll}
\hline Variable & Description \\
\hline \hline$S_{h}(t)$ & Number of susceptible humans at time $t$ \\
$I_{h}(t)$ & Number of infected human at time $t$ \\
$R_{h}(t)$ & Number of recovered humans at time $t$ \\
$S_{c}(t)$ & Number of susceptible cattle at time $t$ \\
$I_{c}(t)$ & Number of infected cattle at time $t$ \\
$V_{c}(t)$ & Number of vaccinated cattle at time $t$ \\
$S_{s}(t)$ & Number of susceptible small ruminants at time $t$ \\
$I_{S}(t)$ & Number of infected small ruminants at time $t$ \\
$V_{s}(t)$ & Number of vaccinated small ruminants at time $t$ \\
$B(t)$ & Number of brucella bacteria load per unit volume in the environment at time $t$ \\
\hline
\end{tabular}

Cattle

Small ruminants

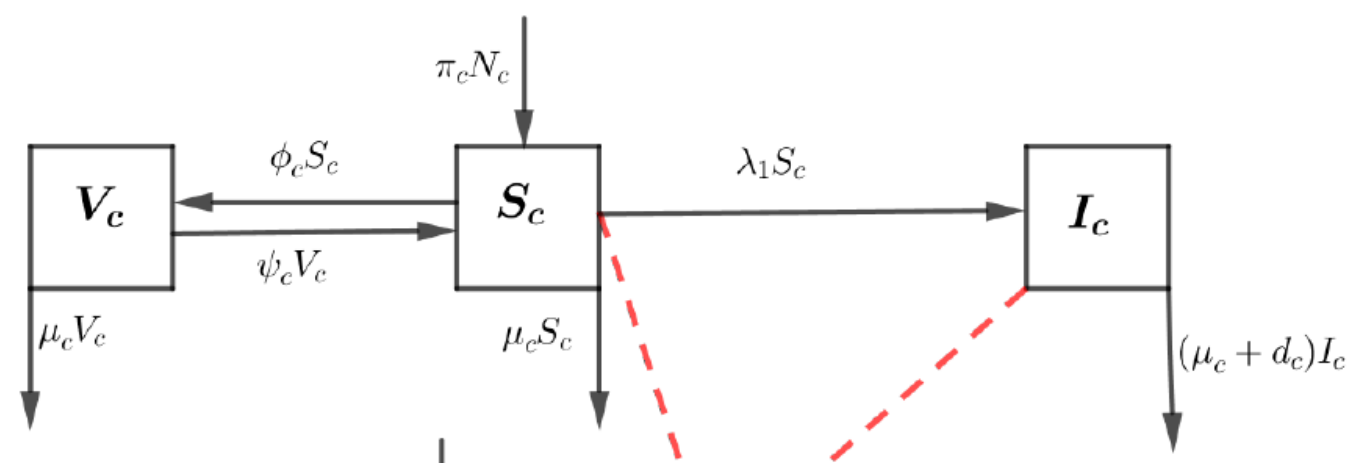

Human

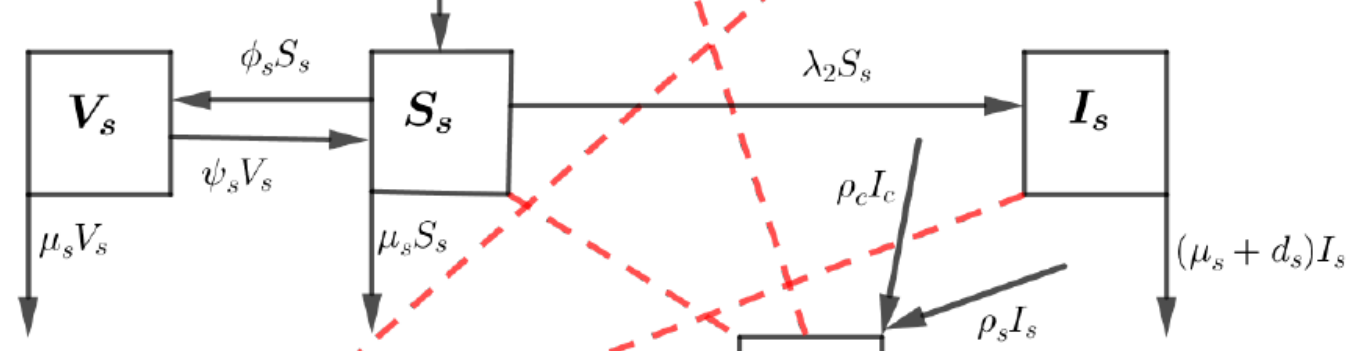

Fig. 1: A schematic diagram for direct and indirect transmission of brucellosis in cattle, small ruminants and human populations. Solid arrows represent transfer of individuals from one subpopulation to another while dotted lines represent interactions leading to infections. 
Table 2: Model Parameters used in the model and their description

\begin{tabular}{ll}
\hline Parameter & Description \\
\hline \hline$\pi_{c}$ & Per capita cattle birth rate \\
$\phi_{c}$ & Cattle vaccination rate \\
$\pi_{h}$ & Per capita human birth rate \\
$\sigma$ & Human recovery rate \\
$\mu_{h}$ & Per capita human natural death rate \\
$\psi_{c}$ & Cattle vaccine efficacy waning rate \\
$\beta_{c}$ & Within cattle transmission rate \\
$d_{c}$ & Culling rate of seropositive cattle \\
$\mu_{c}$ & Per capita cattle natural death rate \\
$\alpha_{c}$ & Brucella from the environment to cattle transmission rate \\
$\alpha_{s}$ & Brucella from the environment to small ruminants transmission rate \\
$\alpha_{h}$ & Brucella from the environment to human transmission rate \\
$\rho_{c}$ & Brucella shedding rate by infected cattle \\
$\rho_{s}$ & Brucella shedding rate by infected small ruminants \\
$\beta_{c h}$ & Cattle to human transmission rate \\
$\beta_{s h}$ & Small ruminants to human transmission rate \\
$\gamma$ & The rate at which recovered human become susceptible \\
$\varepsilon$ & Decaying rate of brucella in the environment \\
$\tau$ & Environmental hygiene and sanitation rate \\
$\pi_{s}$ & Small ruminants per capita birth rate \\
$\phi_{s}$ & Vaccination rate of small ruminants \\
$\psi_{s}$ & Small ruminant vaccine efficacy \\
$\beta_{s}$ & Within small ruminants transmission rate \\
$d_{s}$ & Culling rate of seropositive small ruminants \\
$\mu_{s}$ & Per capita small ruminants natural mortality rate \\
\hline
\end{tabular}

\subsection{Model equations}

Based on the assumptions and the inter-relations between the variables and the parameters as shown in Figure 1, the transmission dynamics of Brucellosis can be described by the following ordinary differential equations:

$$
\begin{aligned}
\frac{d V_{c}}{d t} & =\phi_{c} S_{c}-\left(\mu_{c}+\psi_{c}\right) V_{c} \\
\frac{d S_{c}}{d t} & =\pi_{c} N_{c}+\psi_{c} V_{c}-\left(\lambda_{1}+\phi_{c}+\mu_{c}\right) S_{c} \\
\frac{d I_{c}}{d t} & =\lambda_{1} S_{c}-\left(\mu_{c}+d_{c}\right) I_{c} \\
\frac{d V_{s}}{d t} & =\phi_{s} S_{s}-\left(\mu_{s}+\psi_{s}\right) V_{s} \\
\frac{d S_{s}}{d t} & =\pi_{s} N_{s}+\psi_{s} V_{s}-\left(\lambda_{2}+\phi_{s}+\mu_{s}\right) S_{s} \\
\frac{d I_{s}}{d t} & =\lambda_{2} S_{s}-\left(\mu_{s}+d_{s}\right) I_{s} \\
\frac{d B}{d t} & =\rho_{c} I_{c}+\rho_{s} I_{s}-(\varepsilon+\tau) B \\
\frac{d S_{h}}{d t} & =\pi_{h} N_{h}+\gamma R_{h}-\left(\lambda_{3}+\mu_{h}\right) S_{h}, \\
\frac{d I_{h}}{d t} & =\lambda_{3} S_{h}-\left(\sigma+\mu_{h}\right) I_{h} \\
\frac{d R_{h}}{d t} & =\sigma I_{h}-\left(\gamma+\mu_{h}\right) R_{h}
\end{aligned}
$$


where, $\lambda_{1}=\beta_{c} I_{c}+\alpha_{c} B, \lambda_{2}=\beta_{s} I_{s}+\alpha_{s} B$ and $\lambda_{3}=\beta_{h c} I_{c}+\beta_{h s} I_{s}+\alpha_{h} B$.

\section{Model properties}

Basing on the fact that the first seven equations of system (1) are independent of the last three equations, let us first consider the following model for cattle and the ruminants:

$$
\begin{aligned}
\frac{d V_{c}}{d t} & =\phi_{c} S_{c}-\left(\mu_{c}+\psi_{c}\right) V_{c} \\
\frac{d S_{c}}{d t} & =\pi_{c} N_{c}+\psi_{c} V_{c}-\left(\lambda_{1}+\phi_{c}+\mu_{c}\right) S_{c} \\
\frac{d I_{c}}{d t} & =\lambda_{1} S_{c}-\left(\mu_{c}+d_{c}\right) I_{c} \\
\frac{d V_{s}}{d t} & =\phi_{s} S_{s}-\left(\mu_{s}+\psi_{s}\right) V_{s} \\
\frac{d S_{s}}{d t} & =\pi_{s} N_{s}+\psi_{s} V_{s}-\left(\lambda_{2}+\phi_{s}+\mu_{s}\right) S_{s} \\
\frac{d I_{s}}{d t} & =\lambda_{2} S_{s}-\left(\mu_{s}+d_{s}\right) I_{s} \\
\frac{d B}{d t} & =\rho_{c} I_{c}+\rho_{s} I_{s}-(\varepsilon+\tau) B
\end{aligned}
$$

\subsection{Invariant region}

In this subsection we use Box Invariance method proposed by [1] to assess the well-posedness of the model by investigating the existence and feasibility of its solution. In other words, we investigate whether the solutions are epidemiologically (variables have biological interpretation) and mathematically well-posed (a unique bounded solution exists for all the time). That is solutions of model system (2) with nonnegative initial data remain nonnegative for all time $t \geq 0$. The model system (2) can be expressed in the compact form as:

$$
\frac{d X}{d t}=A(X)+F
$$

where, $X=\left(V_{c}, S_{c}, I_{c}, V_{s}, S_{s}, I_{s}, B\right), F$ is a column vector given by $F=\left(0, \pi_{c} N_{c}, 0,0, \pi_{s} N_{s}, 0,0,0\right)^{T}$ and

$$
A=\left[\begin{array}{ccccccc}
-\left(\mu_{c}+\psi_{c}\right) & \phi_{c} & 0 & 0 & 0 & 0 & 0 \\
\psi_{c} & -\left(\lambda_{1}+\phi_{c}+\mu_{c}\right) & 0 & 0 & 0 & 0 & 0 \\
0 & \lambda_{1} & -\left(\mu_{c}+d_{c}\right) & 0 & 0 & 0 & 0 \\
0 & 0 & 0 & -\left(\mu_{s}+\psi_{s}\right) & \phi_{c} & 0 & 0 \\
0 & 0 & 0 & \psi_{s} & -\left(\lambda_{2}+\phi_{s}+\mu_{s}\right) & 0 & 0 \\
0 & 0 & 0 & 0 & \lambda_{2} & -\left(\mu_{s}+d_{s}\right) & 0 \\
0 & 0 & \rho_{c} & 0 & 0 & \rho_{s} & -(\varepsilon+\tau)
\end{array}\right] .
$$

It can be noticed that $A(X)$ is Meltzer matrix since all of its off diagonal entries are non negative, for all $X \in \mathbb{R}_{+}^{7}$. Therefore, using the fact that $F \geq 0$, the model system (2) is positively invariant in $\mathbb{R}_{+}^{7}$ which means that an arbitrary trajectory of the system starting in $\mathbb{R}_{+}^{7}$ remains in $\mathbb{R}_{+}^{7}$ forever. In addition, the right hand $F$ is Lipschitz continuous. Thus, a unique maximal solution exists and so

$$
\Omega=\left\{\left(V_{c}, S_{c}, I_{c}, V_{s}, S_{s}, I_{s}, B\right) \geq 0\right\} \in \mathbb{R}_{+}^{7},
$$


is the feasible region for the model (2). Thus, the model (2) is epidemiologically and mathematically well-posed in the region $\Omega$.

\section{Model analysis}

\subsection{Disease free equilibrium}

The Brucellosis free equilibrium point is obtained by setting the right hand side of equations in model system (2) to zero, that is:

$$
\frac{d V_{c}}{d t}=\frac{d S_{c}}{d t}=\frac{d I_{c}}{d t}=\frac{d V_{s}}{d t}=\frac{d S_{s}}{d t}=\frac{d I_{s}}{d t}=\frac{d B}{d t}=0 .
$$

Let the disease free equilibrium point of Brucellosis model be $E^{0}$. In case there is no disease $I_{c}=I_{s}=B=0$ that is, the sum of susceptible and vaccinated populations is equal to total population. There exists a disease free equilibrium $E^{0}=\left(V_{c}^{0}, S_{c}^{0}, 0, V_{s}^{0}, S_{s}^{0}, 0,0\right)$ for model system (2) where:

$$
\begin{gathered}
S_{c}^{0}=\frac{\left(\mu_{c}+\psi_{c}\right) \pi_{c} N_{c}^{0}}{\mu_{c}\left(\phi_{c}+\psi_{c}+\mu_{c}\right)}, \quad S_{s}^{0}=\frac{\left(\mu_{s}+\psi_{s}\right) \pi_{s} N_{s}^{0}}{\mu_{s}\left(\phi_{s}+\psi_{s}+\mu_{s}\right)}, \quad V_{c}^{0}=\frac{\phi_{c} \pi_{c} N_{c}^{0}}{\mu_{c}\left(\phi_{c}+\psi_{c}+\mu_{c}\right)}, \\
V_{s}^{0}=\frac{\phi_{s} \pi_{s} N_{s}^{0}}{\mu_{s}\left(\phi_{s}+\psi_{s}+\mu_{s}\right)} .
\end{gathered}
$$

\subsection{The effective reproduction number}

In this subsection, we compute the effective reproduction number for model system (2) using the standard method of the next generation matrix developed in $[17,18]$. The effective reproduction number, $R_{e}$ is defined as the measure of average number of infections caused by a single infectious individual introduced in a community in which intervention strategies are administered [41]. Its magnitude is a useful indicator of both the risk of an epidemic and the effort required to control an infection [58]. When there are no interventions or controls, the number of secondary infections caused by typical infected individual in a completely susceptible population during its entire period of infectiousness is called basic reproduction number, $R_{0}$. It is the threshold parameter to determine whether or not the disease can invade the susceptible population successfully. Due to the natural history of some infections, transmissibility is better quantified by the effective reproduction number rather than the basic reproduction number [15]. Considering the system for the infective variables:

$$
\begin{aligned}
& \frac{d I_{c}}{d t}=\left(\beta_{c} I_{c}+\alpha_{c} B\right) S_{c}-\left(\mu_{c}+d_{c}\right) I_{c}, \\
& \frac{d I_{s}}{d t}=\left(\beta_{s} I_{s}+\alpha_{s} B\right) S_{s}-\left(\mu_{s}+d_{s}\right) I_{s}, \\
& \frac{d B}{d t}=\rho_{c} I_{c}+\rho_{s} I_{s}-(\varepsilon+\tau) B .
\end{aligned}
$$

The effective reproduction number is obtained by taking the spectral radius of the next generation matrix

$$
F V^{-1}=\left[\frac{\partial \mathscr{F}_{i}\left(E^{0}\right)}{\partial t}\right]\left[\frac{\partial \mathscr{V}_{i}\left(E^{0}\right)}{\partial t}\right]^{-1},
$$

where $E^{0}$ is the brucellosis-free equilibrium point while $\mathscr{F}_{i}$ and $\mathscr{V}_{i}$ are vectors representing respectively, the rate of appearance of new infection in compartment $i$ and the transfer of infections from one compartment $i$ to another, such that: 


$$
\begin{gathered}
\mathscr{F}_{i}=\left[\begin{array}{c}
\left(\beta_{c} I_{c}+\alpha_{c} B\right) S_{c} \\
\left(\beta_{s} I_{s}+\alpha_{s} B\right) S_{s} \\
0
\end{array}\right], \\
\mathscr{V}_{i}=\left[\begin{array}{c}
\left(\mu_{c}+d_{c}\right) I_{c} \\
\left(\mu_{s}+d_{s}\right) I_{s} \\
-\rho_{c} I_{c}-\rho_{s} I_{s}+(\varepsilon+\tau) B
\end{array}\right] .
\end{gathered}
$$

It is important to note that $\mathscr{V}_{i}$ is a resultant vector of the two vectors $\mathscr{V}_{i}^{+}$defined as the rate of transfer of individuals into compartment $i$ by all other means, and $\mathscr{V}_{i}^{-}$which is the rate of transfer of individuals out of compartment $i$. That is:

$$
\mathscr{V}_{i}=\mathscr{V}_{i}^{-}-\mathscr{V}_{i}^{+}, i=\{1,2,3\}
$$

The Jacobian matrices $F$ of $\mathscr{F} i$ and $V$ of $\mathscr{V} i$ evaluated at $E^{0}$ are respectively:

$$
F=\left[\begin{array}{ccc}
\beta_{c} S_{c}^{0} & 0 & \alpha_{c} S_{c}^{0} \\
0 & \beta_{s} S_{s}^{0} & \alpha_{s} S_{s}^{0} \\
0 & 0 & 0
\end{array}\right],
$$

and

$$
V=\left[\begin{array}{ccc}
\mu_{c}+d_{c} & 0 & 0 \\
0 & \mu_{s}+d_{s} & 0 \\
-\rho_{c} & -\rho_{s} & (\varepsilon+\tau)
\end{array}\right]
$$

Referring to the infected states with indices $i$ and $j$, for $i, j \in[1,2,3]$, the entry $F_{i j}$ is the rate at which individuals in infected state $j$ give rise or produce new infections to individuals in infected state $i$, in the linearized system. Thus, when there is no new cases produced in infected state $i$ by an individual in infected state $j$ immediately after infection, we have $F_{i j}=0$. The inverse of $V$ is found to be

$$
V^{-1}=\left[\begin{array}{ccc}
\frac{1}{\mu_{c}+d_{c}} & 0 & 0 \\
0 & \frac{1}{\mu_{s}+d_{s}} & 0 \\
\frac{\rho_{c}}{\left(\mu_{c}+d_{c}\right)(\varepsilon+\tau)} & \frac{\rho_{s}}{\left(\mu_{s}+d_{s}\right)(\varepsilon+\tau)} & \frac{1}{\varepsilon+\tau}
\end{array}\right] .
$$

The entry $\left(V^{-1}\right)_{i j}$ is the average length of time an infected individual spends in compartment $j$ during its lifetime when introduced into the compartment $i$ of disease free equilibrium, assuming that the population remains near the disease free equilibrium and barring reinfection. In particular, $\frac{1}{\mu_{c}+d_{c}}$ is an average time an infectious cattle spends in the state of being infective, $\frac{1}{\mu_{s}+d_{s}}$ is the average time spent by an infective small ruminant in the infectious state and $\frac{1}{\varepsilon+\tau}$ is the average time brucella spend in the environment. Furthermore, $\frac{\rho_{c}}{\left(\mu_{c}+d_{c}\right)}$ is the probability that an infective cattle will shed brucella into the environment while $\frac{\rho_{s}}{\left(\mu_{s}+d_{s}\right)}$ is the probability that an infected small ruminant will shed brucella into the environment. Moreover, the Next Generation Matrix is calculated to be:

$$
F V^{-1}=\left[\begin{array}{ccc}
\frac{\beta_{c} S_{c}^{0}}{\mu_{c}+d_{c}}+\frac{\alpha_{c} \rho_{c} S_{c}^{0}}{\left(\mu_{c}+d_{c}\right)(\varepsilon+\tau)} & \frac{\alpha_{c} \rho_{s} S_{c}^{0}}{\left(\mu_{s}+d_{s}\right)(\varepsilon+\tau)} & \frac{\alpha_{c} S_{c}^{0}}{\varepsilon+\tau} \\
\frac{\alpha_{s} \rho_{c} S_{s}^{0}}{\left(\mu_{c}+d_{c}\right)(\varepsilon+\tau)} & \frac{\beta_{s} S_{s}^{0}}{\mu_{s}+d_{s}}+\frac{\alpha_{s} \rho_{s} S_{s}^{0}}{\left(\mu_{s}+d_{s}\right)(\varepsilon+\tau)} & \frac{\alpha_{s} S_{s}^{0}}{\varepsilon+\tau} \\
0 & 0 & 0
\end{array}\right] .
$$


The matrix $F V^{-1}$ can be written as:

$$
F V^{-1}=\left[\begin{array}{ccc}
R_{11} & R_{12} & R_{13} \\
R_{21} & R_{22} & R_{23} \\
0 & 0 & 0
\end{array}\right]
$$

The $(i, k)$ entry of the Next Generation Matrix $F V^{-1}$ is the expected number of secondary infections in compartment $i$ produced by individuals initially in compartment $k$ assuming that the environment seen by the individual remains homogeneous for the duration of its infection [51]. In particular; $R_{11}$ is the expected number of infected cattle produced by one infectious cattle, $R_{12}$ is the expected number of infected cattle produced by one infectious small ruminant via consumption of brucella from the environment, $R_{21}$ is the expected number of infected small ruminant as a result of one infected cattle, and $R_{22}$ is the expected number of infected small ruminant as a result of one infected small ruminant. It can further be noticed that, matrix $F V^{-1}$ is non-negative and therefore, has a nonnegative eigenvalue. The non-negative eigenvalue is associated with a non-negative eigenvector which represents the distribution of infected individuals that produces the greatest number $R_{e}$ of secondary infections per generation [42]. Thus, the spectral radius for our Next Generation Matrix is

$$
\rho\left(F V^{-1}\right)=R_{e}=\frac{R_{11}+R_{22}+\sqrt{\left(R_{22}-R_{11}\right)^{2}+4 R_{12} R_{21}}}{2}
$$

where,

$$
\begin{aligned}
& R_{11}=\frac{\left(\beta_{c}(\varepsilon+\tau)+\alpha_{c} \rho_{c}\right)\left(\psi_{c}+\mu_{c}\right) \pi_{c} N_{c}^{0}}{\mu_{c}\left(\mu_{c}+d_{c}\right)(\varepsilon+\tau)\left(\phi_{c}+\psi_{c}+\mu_{c}\right)}, R_{22}=\frac{\left(\beta_{s}(\varepsilon+\tau)+\alpha_{s} \rho_{s}\right)\left(\psi_{s}+\mu_{s}\right) \pi_{s} N_{s}^{0}}{\mu_{s}\left(\mu_{s}+d_{s}\right)(\varepsilon+\tau)\left(\phi_{s}+\psi_{s}+\mu_{s}\right)} \\
& R_{12}=\frac{\left(\psi_{c}+\mu_{c}\right) \alpha_{c} \rho_{s} \pi_{c} N_{c}^{0}}{\mu_{c}\left(\mu_{s}+d_{s}\right)(\varepsilon+\tau)\left(\phi_{c}+\psi_{c}+\mu_{c}\right)}, R_{21}=\frac{\left(\psi_{s}+\mu_{s}\right) \alpha_{s} \rho_{c} \pi_{s} N_{s}^{0}}{\mu_{s}\left(\mu_{c}+d_{c}\right)(\varepsilon+\tau)\left(\phi_{s}+\psi_{s}+\mu_{s}\right)},
\end{aligned}
$$

When there is no livestock vaccination: $\psi_{c}=\psi_{s}=\phi_{c}=\phi_{s}=0$ and

$$
\begin{gathered}
R_{11}=\frac{\left(\beta_{c}(\varepsilon+\tau)+\alpha_{c} \rho_{c}\right) \pi_{c} N_{c}^{0}}{\mu_{c}\left(\mu_{c}+d_{c}\right)(\varepsilon+\tau)}, \quad R_{22}=\frac{\left(\beta_{s}(\varepsilon+\tau)+\alpha_{s} \rho_{s}\right) \pi_{s} N_{s}^{0}}{\mu_{s}\left(\mu_{s}+d_{s}\right)(\varepsilon+\tau)}, \\
R_{12}=\frac{\alpha_{c} \rho_{s} \pi_{c} N_{c}^{0}}{\mu_{c}\left(\mu_{s}+d_{s}\right)(\varepsilon+\tau)}, \quad R_{21}=\frac{\alpha_{s} \rho_{c} \pi_{s} N_{s}^{0}}{\mu_{s}\left(\mu_{c}+d_{c}\right)(\varepsilon+\tau)},
\end{gathered}
$$

When there is no intervention: $\psi_{c}=\psi_{s}=\phi_{c}=\phi_{s}=\tau=0$, the effective reproduction number becomes the basic reproduction number:

$$
R_{0}=\frac{R_{11}^{0}+R_{22}^{0}+\sqrt{\left(R_{22}^{0}-R_{11}^{0}\right)^{2}+4 R_{12}^{0} R_{21}^{0}}}{2}
$$

where,

$$
R_{11}^{0}=\frac{\left(\beta_{c} \varepsilon+\alpha_{c} \rho_{c}\right) \pi_{c} N_{c}^{0}}{\mu_{c}\left(\mu_{c}+d_{c}\right) \varepsilon}, R_{22}^{0}=\frac{\left(\beta_{s} \varepsilon+\alpha_{s} \rho_{s}\right) \pi_{s} N_{s}^{0}}{\mu_{s}\left(\mu_{s}+d_{s}\right) \varepsilon}, R_{12}^{0}=\frac{\alpha_{c} \rho_{s} \pi_{c} N_{c}^{0}}{\mu_{c}\left(\mu_{s}+d_{s}\right) \varepsilon},
$$

and

$$
R_{21}^{0}=\frac{\alpha_{s} \rho_{c} \pi_{s} N_{s}^{0}}{\mu_{s}\left(\mu_{c}+d_{c}\right) \varepsilon}
$$

In view of the fact that, the first seven equations of model system (1) are independent of the last three equations, system (1) and system (2) have the same effective reproduction and the same basic reproduction number. Thus, the effective reproduction and basic reproduction number for system (1) are $R_{e}$ and $R_{0}$, respectively. 


\subsection{Local stability of the disease free equilibrium}

In this subsection we use the trace-determinant method to investigate the local stability of the brucellosis free equilibrium point.

Theorem 1. The disease free equilibrium for the brucellosis model system(2) is locally asymptotically stable if $R_{0}<1$ and unstable if $R_{0}>1$.

Proof. We show that, variational matrix $J\left(E_{0}\right)$ of the brucellosis model at DFE has a negative trace and positive determinant.

The Jacobian matrix for system 3.2 is given by:

$$
J\left(E_{0}\right)=\left[\begin{array}{ccccccc}
-\left(\mu_{c}+\psi_{c}\right) & \phi_{c} & 0 & 0 & 0 & 0 & 0 \\
\psi_{c} & -\left(\phi_{c}+\mu_{c}\right) & -\beta_{c} S_{c}^{0} & 0 & 0 & 0 & -\alpha_{c} S_{c}^{0} \\
0 & 0 & a_{0} & 0 & 0 & 0 & \alpha_{c} S_{c}^{0} \\
0 & 0 & 0 & -\left(\mu_{s}+\psi_{s}\right) & \phi_{s} & 0 & 0 \\
0 & 0 & 0 & \psi_{s} & -\left(\phi_{s}+\mu_{s}\right) & -\beta_{s} S_{s}^{0} & -\alpha_{s} S_{s}^{0} \\
0 & 0 & 0 & 0 & 0 & a_{1} & \alpha_{s} S_{s}^{0} \\
0 & 0 & \rho_{c} & 0 & 0 & \rho_{s} & -(\varepsilon+\tau)
\end{array}\right]
$$

where,

$$
\begin{aligned}
& a_{0}=\beta_{c} S_{c}^{0}-\left(\mu_{c}+d_{c}\right), \\
& a_{1}=\beta_{s} S_{s}^{0}-\left(\mu_{s}+d_{s}\right) .
\end{aligned}
$$

The trace of the Jacobian matrix $J\left(E_{0}\right)$ is given by:

$$
\begin{aligned}
\operatorname{Tr}\left(J\left(E_{0}\right)\right)= & -\left(\phi_{c}+\psi_{c}+2 \mu_{c}+\varepsilon+\tau+\phi_{s}+\psi_{s}+2 \mu_{s}\right)+\beta_{c} S_{c}^{0}-\left(\mu_{c}+d_{c}\right)+\beta_{s} S_{s}^{0}-\left(\mu_{s}+d_{s}\right), \\
= & -\left(\phi_{c}+\psi_{c}+2 \mu_{c}+\varepsilon+\tau+\phi_{s}+\psi_{s}+2 \mu_{s}\right), \\
& -\left(\mu_{c}+d_{s}\right)\left(1-\frac{\beta_{c} S_{c}^{0}}{\mu_{c}+d_{c}}\right)-\left(\mu_{s}+d_{s}\right)\left(1-\frac{\beta_{s} S_{s}^{0}}{\mu_{s}+d_{s}}\right) .
\end{aligned}
$$

Thus, the trace of the Jocobian matrix is the less than zero, that is $\operatorname{Tr}\left(J\left(E_{0}\right)\right)<0$, if:

On the hand, the determinant of matrix $J\left(E_{0}\right)$ is:

$$
\frac{\beta_{c} S_{c}^{0}}{\mu_{c}+d_{c}}<1 \text { and } \frac{\beta_{s} S_{s}^{0}}{\mu_{c}+d_{s}}<1
$$

$$
\begin{aligned}
\operatorname{Det}\left(J\left(E_{0}\right)\right)= & \mu_{c} \mu_{s}\left(\phi_{c}+\psi_{c}+\mu_{c}\right)\left(\phi_{s}+\psi_{s}+\mu_{s}\right)\left[\left(\mu_{s}+d_{s}\right)(\varepsilon+\tau) \beta_{c} S_{c}^{0}\left(1-\frac{\beta_{s} S_{s}^{0}}{\mu_{s}+d_{s}}\right)\right. \\
& +\left(\mu_{c}+d_{c}\right) \rho_{s} \alpha_{s} S_{s}^{0}\left(1-\frac{\beta_{c} S_{c}^{0}}{\mu_{c}+d_{c}}\right)+\left(\mu_{s}+d_{s}\right) \rho_{c} \alpha_{c} S_{c}^{0}\left(1-\frac{\beta_{s} S_{s}^{0}}{\mu_{s}+d_{s}}\right) \\
& \left.-\left(\mu_{c}+d_{c}\right)\left(\mu_{s}+d_{s}\right)(\varepsilon+\tau)\left(1-\frac{\beta_{s} S_{s}^{0}}{\mu_{s}+d_{s}}\right)\right], \\
= & \mu_{c} \mu_{s}\left(\phi_{c}+\psi_{c}+\mu_{c}\right)\left(\phi_{s}+\psi_{s}+\mu_{s}\right)\left(\mu_{c}+d_{c}\right)\left(\mu_{s}+d_{s}\right)(\varepsilon+\tau) \\
& \left(\frac{\alpha_{c} \rho_{c} S_{c}^{0}\left(1-\frac{\beta_{s} S_{s}^{0}}{\mu_{s}+d_{s}}\right)}{(\varepsilon+\tau)\left(\mu_{s}+d_{s}\right)}-\left(1-\frac{\beta_{c} S_{c}^{0}}{\mu_{c}+d_{c}}\right)\left(1-\frac{\left(\beta_{s}(\varepsilon+\tau)+\alpha_{s} \rho_{s}\right) S_{s}^{0}}{(\varepsilon+\tau)\left(\mu_{s}+d_{s}\right)}\right)\right) .
\end{aligned}
$$


The determinant of the Jacobian matrix is positive (i.e. $J\left(E_{0}\right)>0$ ) iff:

$$
\begin{gathered}
\left(\frac{\alpha_{c} \rho_{c} S_{c}^{0}\left(1-\frac{\beta_{s} S_{s}^{0}}{\mu_{s}+d_{s}}\right)}{(\varepsilon+\tau)\left(\mu_{s}+d_{s}\right)}>\left(1-\frac{\beta_{c} S_{c}^{0}}{\mu_{c}+d_{c}}\right)\left(1-\frac{\left(\beta_{s}(\varepsilon+\tau)+\alpha_{s} \rho_{s}\right) S_{s}^{0}}{(\varepsilon+\tau)\left(\mu_{s}+d_{s}\right)}\right)\right), \\
\frac{\beta_{c} S_{c}^{0}}{\mu_{c}+d_{c}}<1, \quad \frac{\beta_{s} S_{s}^{0}}{\mu_{c}+d_{s}}<1,
\end{gathered}
$$

and

$$
\frac{\left((\varepsilon+\tau) \beta_{c}+\rho_{c} \alpha_{c}\right) S_{c}^{0}}{(\varepsilon+\tau)\left(\mu_{c}+d_{c}\right)}<1 .
$$

Furthermore, $\frac{\beta_{c} S_{c}^{0}}{\mu_{c}+d_{c}}$ and $\frac{\beta_{s} S_{s}^{0}}{\mu_{c}+d_{s}}$ are respectively the average number of cattle infections as a result of direct contact between susceptible and infected cattle and the average number of small ruminant infections as a result of direct contact between susceptible and infected small ruminant, and $\frac{\left((\varepsilon+\tau) \beta_{c}+\rho_{c} \alpha_{c}\right) S_{c}^{0}}{(\varepsilon+\tau)\left(\mu_{c}+d_{c}\right)}$ is the expected number of infected cattle caused directly or indirectly by one infectious cattle. Thus, the brucellosis free equilibrium for each population is locally asymptotically stable if and only if the number of secondary infections, $\left(R_{e}\right)$ is less than unit, that is $R_{0}<1$. This completes the proof.

\subsection{Global stability of the disease-free equilibrium}

In this section, we analyze the global stability of the disease-free equilibrium point by applying the [11] approach. We write model system (2) in the form:

$$
\left\{\begin{array}{l}
\frac{d X_{s}}{d t}=A\left(X_{s}-X_{D F E, S}\right)+A_{1} X_{i} \\
\frac{d X_{i}}{d t}=A_{2} X_{i}
\end{array}\right.
$$

where $X_{s}$ is the vector representing the non-transmitting compartments and $X_{i}$ is the vector representing the transmitting components. The DFE is globally asymptotically stable if $\mathrm{A}$ has real negative eigenvalues and $A_{2}$ is a Metzler matrix (i.e. the off-diagonal elements of $A_{2}$ are non-negative). From model system (2) we have:

$$
X_{i}=\left(I_{c}, I_{s}, B\right)^{T}, X_{s}=\left(V_{c}, S_{c}, V_{s}, S_{s}\right)^{T}, X_{s}-X_{D F E, s}=\left[\begin{array}{c}
V_{c}-\frac{\phi_{c} \pi_{c} N_{c}^{0}}{\mu_{c}\left(\phi_{c}+\mu_{c}+\psi_{c}\right)+\psi_{c}} \\
S_{c}-\frac{\left(\phi_{c}+\mu_{c}\right) \pi_{c} N_{c}^{0}}{\mu_{c}\left(\phi_{c}+\mu_{c}+\psi_{c}\right)+\psi_{c}} \\
\phi_{s} \pi_{s} N_{s}^{0} \\
V_{s}-\frac{\psi_{s}\left(\phi_{s}+\mu_{s}+\psi_{s}\right)+\psi_{s}}{\mu_{s}} \\
S_{s}-\frac{\left(\phi_{s}+\mu_{s}\right) \pi_{s} N_{s}^{0}}{\mu_{s}\left(\phi_{s}+\mu_{s}+\psi_{s}\right)+\psi_{s}}
\end{array}\right],
$$

and

$$
A_{1}=\left[\begin{array}{ccc}
0 & 0 & 0 \\
-\beta_{c} S_{c} & 0 & -\alpha_{c} \\
0 & 0 & 0 \\
0 & -\beta_{s} S_{s}-\alpha_{s}
\end{array}\right]
$$


We need to check whether a matrix $A$ for the non-transmitting compartments has real negative eigenvalues and that $A_{2}$ is a Metzler matrix. From the equation for non-transmitting compartments in (2) we have:

$$
A=\left[\begin{array}{cccc}
-\left(\psi_{c}+\mu_{c}\right) & \phi_{c} & 0 & 0 \\
\psi_{c} & -\left(\phi_{c}+\mu_{c}\right) & 0 & 0 \\
0 & 0 & -\left(\psi_{s}+\mu_{s}\right) & \phi_{s} \\
0 & 0 & \psi_{s} & -\left(\phi_{s}+\mu_{s}\right)
\end{array}\right]
$$

with eigenvalues $\lambda_{1}=-\mu_{s}, \lambda_{2}=-\left(\psi_{s}+\phi_{s}+\mu_{s}\right), \lambda_{3}=-\mu_{c}, \lambda_{4}=-\left(\psi_{c}+\phi_{c}+\mu_{c}\right)$ and

$$
A_{2}=\left[\begin{array}{ccc}
\beta_{c} S_{c}^{0}-\left(\mu_{c}+d_{c}\right) & 0 & \alpha_{c} S_{c}^{0} \\
0 & \beta_{s} S_{s}^{0}-\left(\mu_{s}+d_{s}\right) & \alpha_{s} S_{s}^{0} \\
\rho_{c} & \rho_{s} & -(\varepsilon+\tau)
\end{array}\right]
$$

It can be seen that, $A_{2}$ which is a Metzler matrix, and $A$, have real negative eigenvalues. This implies that the disease free equilibrium for the model system (2) is globally asymptotically stable.

\subsection{Global stability of endemic equilibrium}

The local stability of the disease free equilibrium suggests local stability of the endemic equilibrium for the reverse condition $[9,10,51]$. In this subsection we study the global behaviour of the endemic equilibrium, $E^{*}$ for the model system (2).

Theorem 2. The endemic equilibrium point for the brucellosis model system (2) is globally asymptotically stable on $\Omega$ if $R_{0}>1$.

Proof. We construction an explicit Lyapunov function for model system (2) using [7,30,31,32,36] approach as it is useful to most of the sophisticated compartmental epidemiological models. In this approach, we construct Lyapunov function of the form:

$$
V=\sum a_{i}\left(x_{i}-x_{i}^{*} \ln x\right)
$$

where $a_{i}$ is a properly selected positive constant, $x_{i}$ is the population of the $i^{t h}$ compartment and $x_{i}^{*}$ is the equilibrium level. We define the Lyapunov function candidate $V$ for model system (2) as:

$$
\begin{aligned}
L= & \left(S_{c}-S_{c}^{*} \ln S_{c}\right)+A_{1}\left(V_{c}-V_{c}^{*} \ln V_{c}\right)+A_{2}\left(I_{c}-I_{c}^{*} \ln I_{c}\right)+\left(S_{s}-S_{s}^{*} \ln S_{s}\right) \\
& +A_{3}\left(V_{s}-V_{s}^{*} \ln V_{s}\right)+A_{4}\left(I_{s}-I_{s}^{*} \ln I_{s}\right)+A_{5}\left(B-B^{*} \ln B\right),
\end{aligned}
$$

where $A_{1}, A_{2}, A_{3}, A_{4}$ and $A_{5}$ are positive constants. The time derivative of the Lyapunov function $L$ is given by:

$$
\begin{aligned}
\frac{d L}{d t}= & \left(1-\frac{S_{c}^{*}}{S_{c}}\right) \frac{d S_{c}}{d t}+A_{1}\left(1-\frac{V_{c}^{*}}{V_{c}}\right) \frac{d V_{c}}{d t}+A_{2}\left(1-\frac{I_{c}^{*}}{I_{c}}\right) \frac{d I_{c}}{d t}+\left(1-\frac{S_{s}^{*}}{S_{s}}\right) \frac{d S_{s}}{d t} \\
& +A_{3}\left(1-\frac{V_{s}^{*}}{V_{s}}\right) \frac{d V_{s}}{d t}+A_{4}\left(1-\frac{I_{s}^{*}}{I_{s}}\right) \frac{d I_{s}}{d t}+A_{5}\left(1-\frac{B^{*}}{B}\right) \frac{d B}{d t} .
\end{aligned}
$$


Considering (2) at $E^{*}$ we have:

$$
\begin{gathered}
\phi=\frac{\left(\psi_{c}+\mu_{c}\right) V_{c}^{*}}{S_{c}^{*}}, \\
\pi_{c} N_{c}^{*}=\left(\beta_{c} I_{c}^{*}+\alpha_{c} B^{*}+\phi_{c}+\mu_{c}\right) S^{*}-\psi_{c} V_{c}^{*}, \\
\mu_{c}+d_{c}=\frac{\left(\beta_{c} I_{c}^{*}+\alpha_{c} B^{*}\right) S^{*}}{I_{c}^{*}}, \\
(\varepsilon+\tau)=\frac{\rho_{c} I_{c}^{*}+\rho_{s} I_{s}^{*}}{I_{c}^{*}} .
\end{gathered}
$$

Then, equation (8) may be re-written as:

$$
\begin{aligned}
\frac{d L}{d t}= & -\left(\phi_{c}+\mu_{c}\right) S_{c}\left(1-\frac{S_{c}^{*}}{S_{c}}\right)^{2}-\left(\phi_{s}+\mu_{s}\right) S_{s}\left(1-\frac{S_{s}^{*}}{S_{s}}\right)^{2} \\
& -\left(1-\frac{S_{c}^{*}}{S_{c}}\right)\left(\beta_{c} I_{c} S_{c}\left(1-\frac{I_{c}^{*} S_{c}^{*}}{I_{c} S_{c}}\right)+\alpha_{c} B S_{c}\left(1-\frac{B^{*} S_{c}^{*}}{B S_{c}}\right)+\psi_{c} V_{c}\left(\frac{V_{c}^{*}}{V_{c}}-1\right)\right) \\
& -\left(1-\frac{S_{s}^{*}}{S_{s}}\right)\left(\beta_{s} I_{s} S_{s}\left(1-\frac{I_{s}^{*} S_{s}^{*}}{I_{s} S_{s}}\right)+\alpha_{s} B S_{s}\left(1-\frac{B^{*} S_{s}^{*}}{B S_{s}}\right)+\psi_{s} V_{s}\left(\frac{V_{s}^{*}}{V_{s}}-1\right)\right) \\
& -\left(\psi_{c}+\mu_{c}\right) B V_{c} A_{1}\left(1-\frac{V_{c}^{*}}{V_{c}}\right)\left(1-\frac{V_{c}^{*}}{V_{c} S_{c}^{*}}\right)-\left(\psi_{s}+\mu_{s}\right) B V_{s} A_{3}\left(1-\frac{V_{s}^{*}}{V_{s}}\right)\left(1-\frac{V_{s}^{*}}{V_{s} S_{s}^{*}}\right) \\
& +A_{2}\left(1-\frac{I_{c}^{*}}{I_{c}}\right)\left(\beta_{c} I_{c} S_{c}\left(1-\frac{S_{c}^{*}}{S_{c}}\right)+\alpha_{c} B S_{c}\left(1-\frac{B^{*} S_{c}^{*} I_{c}}{B S_{c} I_{c}^{*}}\right)\right) \\
& +A_{4}\left(1-\frac{I_{s}^{*}}{I_{s}}\right)\left(\beta_{s} I_{s} S_{s}\left(1-\frac{S_{s}^{*}}{S_{s}}\right)+\alpha_{s} B S_{s}\left(1-\frac{B^{*} S_{s}^{*} I_{s}}{B S_{S} I_{s}^{*}}\right)\right) \\
& +A_{5}\left(1-\frac{B^{*}}{B}\right)\left(\rho_{c} I_{c}\left(1-\frac{B I_{c}^{*}}{B^{*} I_{c}}\right)+\rho_{s} I_{s}\left(1-\frac{B I_{s}^{*}}{B^{*} I_{s}}\right)\right) .
\end{aligned}
$$

Equation (9) can be written as:

$$
\frac{d L}{d t}=-\left(\left(\phi_{c}+\mu_{c}\right) S_{c}\left(1-\frac{S_{c}^{*}}{S_{c}}\right)^{2}+\left(\phi_{s}+\mu_{s}\right) S_{s}\left(1-\frac{S_{s}^{*}}{S_{s}}\right)^{2}\right)+F\left(S_{c}, V_{c}, I_{c}, S_{s}, V_{s}, I_{s}, B\right),
$$

where $F$ is the balance of the right hand terms of equation (9). Following the approach of $[7,30,31,32,33,36], F$ is a non-positive function for $S_{c}, V_{c}, I_{c}, S_{s}, V_{s}, I_{s}, B>0$. Thus, $\frac{d L}{d t}<0$ for $S_{c}, V_{c}, I_{c}, S_{s}, V_{s}, I_{s}, B>0$ and is zero if $S_{c}=S_{c}^{*}, V_{c}=$ $V_{c}^{*}, I_{c}=I_{c}^{*}, S_{s}=S_{s}^{*}, V_{s}=V_{s}^{*}, I_{s}=I_{s}^{*}$, and $B=B^{*}$. Therefore, if $R_{e}>1$, model system (2) has a an endemic equilibrium point $E^{*}$ which is locally and globally asymptotically stable.

\section{Sensitivity analysis}

In this section, we investigate the relative importance of the parameters featuring in the effective reproduction number. Brucellosis incidences and prevalences can best be reduced or eradicated if the parameters with significant impact in the transmission dynamics of the disease are taken into consideration when planning for and implementing intervention strategies. Sensitivity analysis is commonly used to determine the robustness of model predictions to parameter values, since there are usually errors in data collection and presumed parameter values [12]. Sensitivity indices provide information on how vital each parameter is to disease transmission and prevalence, and permits measurement of relative changes in a state variable when a parameter changes. Thus, we use sensitivity analysis to discover parameters that have 
high impact on the reproduction number, $R_{e}$ and that should be targeted by intervention strategies. We know that initial disease transmission is directly related to $R_{e}$, therefore we compute the sensitivity indices of $R_{e}$ for the parameters in model 2. The explicit expression of $R_{e}$ is given by equation 4 . Since $R_{e}$ depends only on twenty parameters, we derive an analytical expression for its sensitivity to each parameter using the normalized forward sensitivity index [35] as follows:

$$
\begin{aligned}
& \Upsilon_{\mu_{c}}^{R_{e}}=\frac{\partial R_{e}}{\partial \mu_{c}} \times \frac{\mu_{c}}{R_{e}}=-0.84, \\
& \Upsilon_{\pi_{c}}^{R_{e}}=\frac{\partial R_{e}}{\partial \pi_{c}} \times \frac{\pi_{c}}{R_{e}}=+0.69 .
\end{aligned}
$$

In a similar fashion, we compute the sensitivity indices for all parameters used in equation 4 and present the results in Table 3. Table 3 shows that the most sensitive parameters of the effective reproductive number in each population are natural

Table 3: Sensitivity indices for $R_{e}$ parameters

\begin{tabular}{llll}
\hline Parameter & Value & Unit & Sensitivity Index \\
\hline \hline$\pi_{c}$ & 0.3 & year $^{-1}$ & 0.69 \\
$\beta_{c}$ & 0.0011 & year $^{-1}$ & 0.54 \\
$\phi_{c}$ & 0.7 & year $^{-1}$ & -0.36 \\
$\psi_{c}$ & 0.4 & year $^{-1}$ & 0.22 \\
$\mu_{c}$ & 0.25 & year $^{-1}$ & -0.84 \\
$d_{c}$ & 0.35 & year $^{-1}$ & -0.40 \\
$\alpha_{c}$ & 0.00035 & year $^{-1}$ & 0.15 \\
$\rho_{c}$ & 10 & year $^{-1}$ & 0.15 \\
$\varepsilon$ & 8 & year $^{-1}$ & -0.10 \\
$\tau$ & 12 & year $^{-1}$ & -0.16 \\
$\pi_{s}$ & 0.4 & year $^{-1}$ & 0.31 \\
$\beta_{s}$ & 0.001 & year $^{-1}$ & 0.20 \\
$\phi_{s}$ & 0.8 & year $^{-1}$ & -0.15 \\
$\psi_{s}$ & 0.5 & year $^{-1}$ & 0.09 \\
$\mu_{s}$ & 0.35 & year $^{-1}$ & -0.39 \\
$d_{s}$ & 0.4 & year $^{-1}$ & -0.16 \\
$\alpha_{s}$ & 0.00032 & year $^{-1}$ & 0.11 \\
$\rho_{s}$ & 15 & year $^{-1}$ & 0.11 \\
\hline
\end{tabular}

death rate, birth rate, transmission rate, gradual culling rate of sero-positive ruminants through slaughter and vaccination rate. The positive sign in the sensitivity index means that an increase in that parameter leads to an increase in $R_{e}$ and vise-versa. For instance, an increase or decrease of cattle birth rate by $10 \%$ leads to an increase or decrease of $R_{e}$ by $6.9 \%$. On the other hand, the negative sign in the sensitivity index of a parameter indicates that an increase or decrease in a parameter value leads to a decrease or increase in $R_{e}$ respectively. For instance, a $10 \%$ increase in cattle natural mortality rate leads to a $8.4 \%$ decrease in the effective reproductive number. This implies that culling in large livestock flocks is inevitable if we want to control brucellosis transmissions.

\section{Numerical Simulations}

This section presents numerical simulations for model system 1 for the purpose of verifying some of the analytical results. The parameter values used in our computations are mainly from [34], a literature similar to this work. The parameter values are in Table 3. Figure 2 illustrates the variations in livestock and brucella subpopulations as time increases. Figure 2 shows that susceptible ruminants decrease rapidly due to brucellosis epidemic and vaccination of susceptible ruminants, while the infective subpopulations initially increase with time. However, after a two-year period these subpopulations start decreasing. The increase in the infective classes is due to high brucellosis transmission rate 


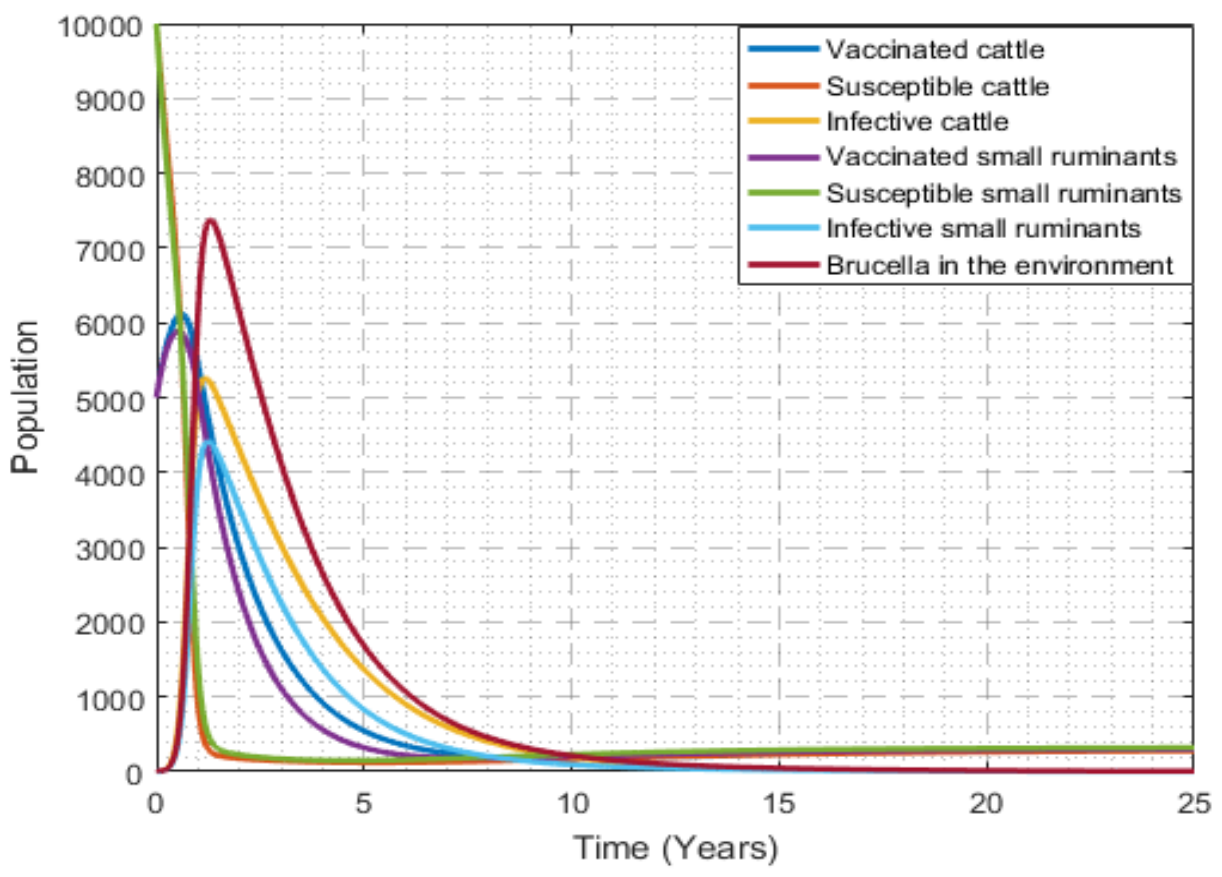

Fig. 2: Ruminants subpopulations variation

and the decrease is due to different interventions like gradual culling of infective ruminants, environmental hygiene and sanitation, and immunization of susceptible ruminants. The graph for vaccinated classes initially increase because large number of susceptible livestock are vaccinated at the beginning of any vaccination program and decrease due to reduction in the number of susceptible livestock. Furthermore, the number of all infective classes goes to zero after 10 years. From Figure 3 we see that the combination of timely environmental hygiene and sanitation and ruminants vaccination significantly controls the indirect transmission of brucella from cattle to small ruminants and vice versa. In addition, the disease can be eliminated from the population if gradual culling of seropositive ruminants through slaughter eliminates at least $35 \%$ and $40 \%$ of the infective cattle and small ruminants respectively.

Furthermore, Figure 4a shows that an increase in cattle vaccination rate leads to a decrease in the effective reproduction number. For instance, the cattle population attains its disease free equilibrium at $10 \%$ vaccination rate provided that other controls are kept constant. This implies that cattle vaccination at some points plays a significant contribution in reducing the transmission dynamics of brucellosis. A similar trend is observed from Figure $4 \mathrm{~b}$ that vaccination of small ruminants significantly reduces their secondary brucellosis infections and the small ruminants brucellosis free state is achieved at 9\% vaccination rate. Moreover, if other control parameters are kept constant and disease-induced rate is varied, we obtain the brucellosis free equilibrium $\left(R_{e}<1\right)$ at $12.5 \%$ and $10 \%$ diseased induced elimination rates for cattle and small ruminants respectively (see Figure 5a and Figure $5 \mathrm{~b}$ ).

Generally, the combination of ruminants vaccination, test-and-slaughter and disinfection of the environment minimizes or eliminates the disease from the populations. In line with this [47] pointed out that, gradual culling of seropositive animals through slaughter, isolation and confinement of pregnant cows close to calving; proper disposal of placentas and aborted foetuses, the use of the S19 vaccine, and restricted introduction of new animals leads to brucellosis elimination in animal herds. 


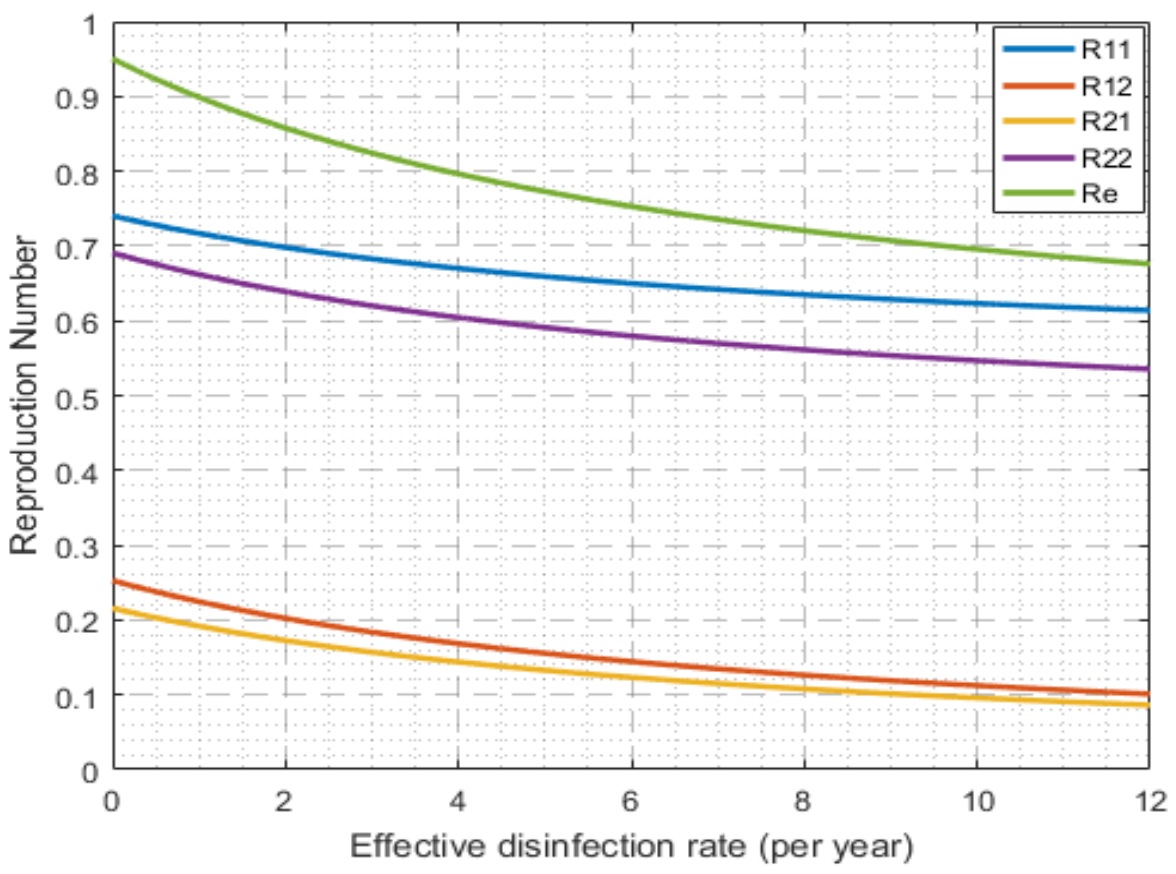

Fig. 3: The impact of environmental hygiene and sanitation on brucelosis transmission

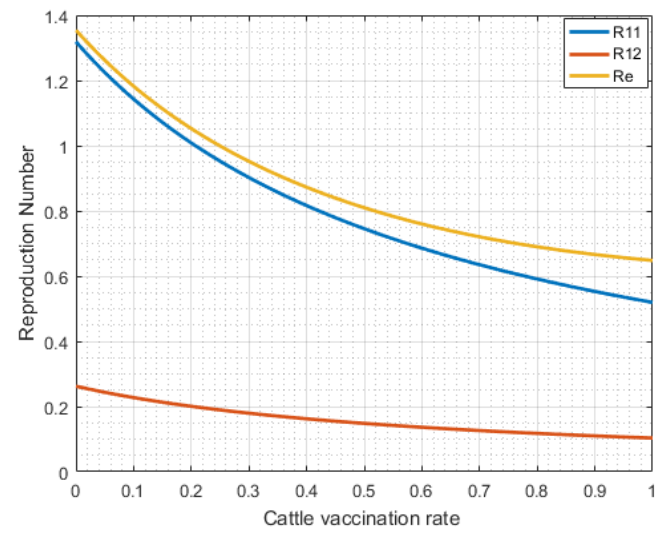

(a)

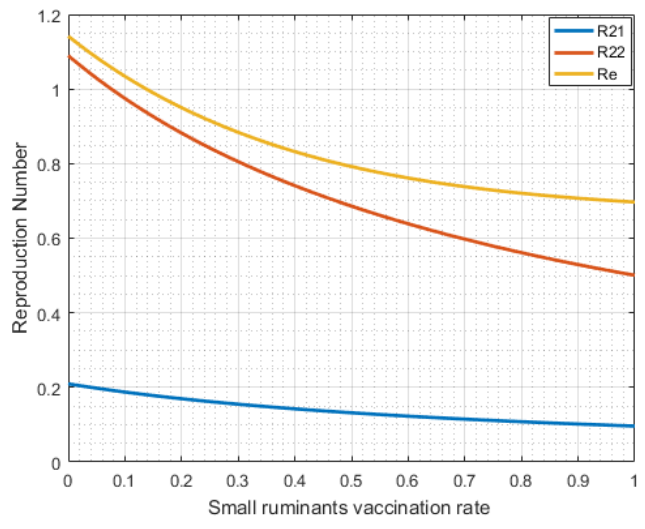

(b)

Fig. 4: The impact of ruminants vaccination on brucelosis transmission.

\section{Conflict of Interest}

The authors declares no conflict of interest regarding the publication of this manuscript.

\section{Authors' contributions}

All authors have contributed to all parts of the article. All authors read and approved the final manuscript. 


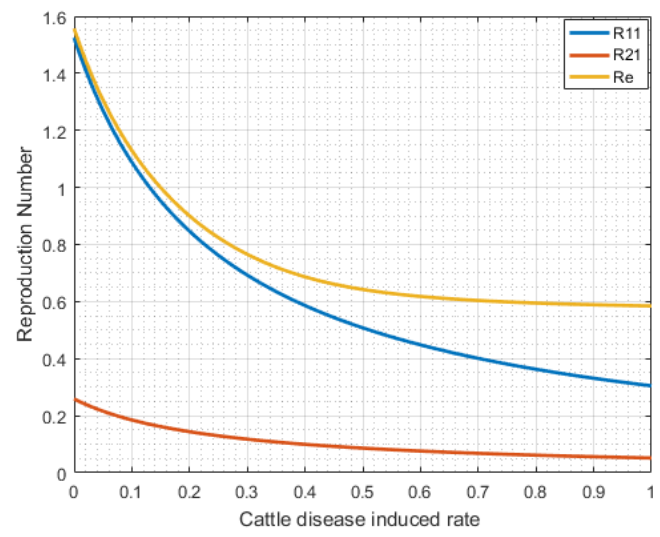

(a)

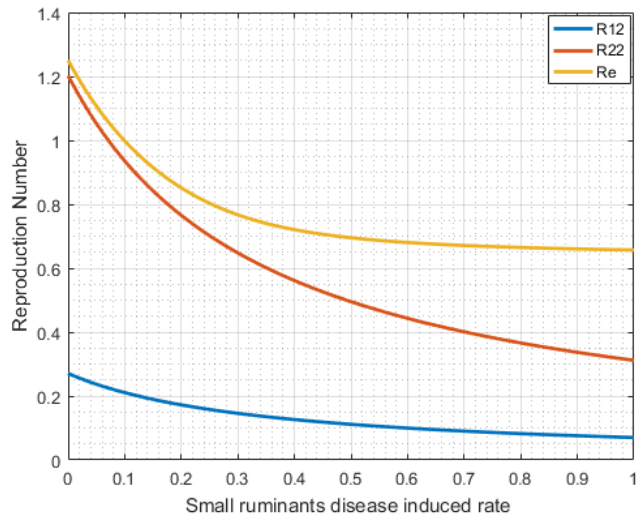

(b)

Fig. 5: The impact of seropositive ruminants culling on the transmission of brucellosis.

\section{References}

[1] Alessandro Abate, Ashish Tiwari, and Shankar Sastry. Box invariance in biologically-inspired dynamical systems, Automatica; 45(9): 1601-1610, 2009.

[2] Bedr'Eddine Ainseba, Chahrazed Benosman, and Pierre Magal. A model for ovine brucellosis incorporating direct and indirect transmission, Journal of biological dynamics; 4(1): 2-11, 2010.

[3] Ali G Alhamada, Ihab Habib, Anne Barnes, and Ian Robertson. Risk factors associated with brucella seropositivity in sheep and goats in Duhok Province, Iraq, Veterinary sciences,4(65): 1-9, 2017.

[4] M Amaku, RA Dias, JS Ferreira Neto, and F Ferreira. Mathematical modeling of bovine brucellosis control by vaccination, Arquivo Brasileiro de Medicina Veterinária e Zootecnia, 61:135-141, 2009.

[5] J Awah-Ndukum, MMM Mouiche, HN Bayang, V Ngu Ngwa, E Assana, KJM Feussom, TK Manchang, and PA Zoli. Seroprevalence and Associated Risk Factors of Brucellosis among Indigenous Cattle in the Adamawa and North Regions of Cameroon, Veterinary medicine international, 2018. URL:https://doi.org/10.1155/2018/3468596.

[6] Andrew J Bouley, Holly M Biggs, Robyn A Stoddard, Anne B Morrissey, John A Bartlett, Isaac A Afwamba, Venance P Maro, Grace D Kinabo, Wilbrod Saganda, Sarah Cleaveland, and John A Crump. Brucellosis Among Hospitalized Febrile Patients in Northern Tanzania, The American journal of tropical medicine and hygiene, 87(6):1105-1111, 2012.

[7] Samuel Bowong, Jean Jules Tewa, and Jean Claude Kamgang. Stability analysis of the transmission dynamics of tuberculosis models, World Journal of Modelling and Simulation; 7(2): 83-100, 2011.

[8] Manuela Carugati, Holly M Biggs, Michael J Maze, Robyn A Stoddard, Shama Cash-Goldwasser, Julian T Hertz, Jo EB Halliday, Wilbrod Saganda, Bingileki F Lwezaula, Rudovick R Kazwala, Sarah Cleaveland, Venance P Maro, Mathew P Rubach, and John A Crump. Incidence of human brucellosis in the Kilimanjaro Region of Tanzania in the periods 2007-2008 and 2012-2014, Transactions of The Royal Society of Tropical Medicine and Hygiene; 112(3): 136-143, 2018.

[9] Saul C Mpeshe, Livingstone S Luboobi, and Yaw Nkansah-Gyekye. Stability analysis of the Rift Valley fever dynamical model,Journal of Mathematical and Computational Science, 4(4): 740-762, 2014.

[10] N Nyerere, LS Luboobi, and Y Nkansah-Gyekye. Bifurcation and Stability analysis of the dynamics of Tuberculosis model incorporating, vaccination, Screening and treatment,Communications in Mathematical biology and Neuroscience, 1:Article-ID, 2014.

[11] Carlos Castillo-Chavez, Sally Blower, Pauline van den Driessche, Denise Kirschner, and Abdul-AzizYakubu. Mathematical approaches for emerging and reemerging infectious diseases: an introduction, 1: 2002.

[12] Nakul Chitnis, James M Hyman, and Jim M Cushing. Determining important parameters in the spread of malaria through the sensitivity analysis of a mathematical model, Bulletin of mathematical biology, 70(5): 1272, 2008. 
[13] CDC. Brucellosis Signs and Symptoms, https://www.cdc.gov/brucellosis/symptoms/index.html. Accessed 2018-11-07.

[14] CFSPH. Brucellosis Brucella abortus, http://cfsph.iastate.edu/Factsheets/pdfs/brucellosis_abortus.pdf. Accessed 2018-11-07.

[15] Ariel Cintrón-Arias, Carlos Castillo-Chávez, Luıs MA Bettencourt, Alun L Lloyd, and HT Banks. The estimation of the effective reproductive number from disease outbreak data, Math Biosci Eng, 6(2): 261-282, 2009.

[16] Anna S Dean, Lisa Crump, Helena Greter, Esther Schelling, and Jakob Zinsstag. Global burden of human brucellosis: a systematic review of disease frequency, PLoS neglected tropical diseases, 6(10): 1-9, 2012.

[17] Odo Diekmann, Johan Andre Peter Heesterbeek, and Johan AJ Metz. On the definition and the computation of the basic reproduction ratio $R_{0}$ in models for infectious diseases in heterogeneous populations,Journal of mathematical biology, 28(4): 365-382, 1990.

[18] O Diekmann, JAP Heesterbeek, and MG Roberts. The construction of next-generation matrices for compartmental epidemic models, Journal of the Royal Society Interface, 7(47): 873-885, 2010.

[19] Andrew Dobson, and Mary Meagher. The population dynamics of brucellosis in the Yellowstone National Park, Ecology, 77(4): 1026-1036, 1996.

[20] M Ducrotoy, WJ Bertu, G Matope, S Cadmus, R Conde-Álvarez, AM Gusi, S Welburn, R Ocholi, JM Blasco, and I Moriyón. Brucellosis in Sub-Saharan Africa: current challenges for management, diagnosis and control, Acta tropica, 165: 179-193, 2017.

[21] KA Franc, RC Krecek, BN Häsler, and AM Arenas-Gamboa. Brucellosis remains a neglected disease in the developing world, BMC public health, 18(125): 2018.

[22] M Graeme Garner, and SD Beckett . Modelling the spread of foot-and-mouth disease in Australia, Australian Veterinary Journal, 83(12): 758-766, 2005.

[23] Giraldo J Ospina and Palacio D Hincapié. Deterministic SIR (Susceptible-Infected-Removed) models applied to varicella outbreaks, Epidemiology \& Infection, 136(5): 679-687, 2008.

[24] Ronald A Greenfield, Douglas A Drevets, Linda J Machado, Gene W Voskuhl, Paul Cornea, and Michael S Bronze. Bacterial pathogens as biological weapons and agents of bioterrorism, The American journal of the medical sciences, 323(6): 299-315, 2002.

[25] Qiang Hou, Xiangdong Sun, Juan Zhang, Yongjun Liu, Youming Wang, and Zhen Jin. Modeling the transmission dynamics of sheep brucellosis in Inner Mongolia Autonomous Region, China, Mathematical biosciences, 242(1): 51-58, 2013.

[26] Qiang Hou, and Xiang-Dong Sun. Modeling sheep brucellosis transmission with a multi-stage model in Changling County of Jilin Province, China, Journal of Applied Mathematics and Computing, 51(1-2): 227-244, 2016.

[27] Kunda John, Julie Fitzpatrick, Nigel French, Rudovick Kazwala, Dominic Kambarage, Godfrey S Mfinanga, Alastair MacMillan, and Sarah Cleaveland. Quantifying risk factors for human brucellosis in rural northern Tanzania, PloS one, 5(4): 1-6, 2010.

[28] Mirjam Sarah Kadelka. Mathematical models of immune responses following vaccination with application to Brucella infection , Virginia Tech, 242(1): 2015.

[29] MJ Keeling, and L Danon. Mathematical modelling of infectious diseases, British Medical Bulletin, 92(1): 33-42, 2009.

[30] Andrei Korobeinikov, and Graeme C Wake. Lyapunov functions and global stability for SIR, SIRS, and SIS epidemiological models, Applied Mathematics Letters, 15(8): 955-960, 2002.

[31] Andrei Korobeinikov. Lyapunov functions and global properties for SEIR and SEIS epidemic models, Mathematical medicine and biology: a journal of the IMA, 21(2): 75-83, 2004.

[32] Andrei Korobeinikov. Global properties of infectious disease models with nonlinear incidence, Bulletin of Mathematical Biology, 69(6): 1871-1886, 2007.

[33] Can Li, Zun-Guang Guo, and Zhi-Yu Zhang. Transmission dynamics of a brucellosis model: Basic reproduction number and global analysis, Chaos, Solitons \& Fractals, 104: 161-172, 2017.

[34] Ming-Tao Li, Gui-Quan and Sun, Yan-Fang Wu, Juan Zhang, and Zhen Jin. Transmission dynamics of a multi-group brucellosis model with mixed cross infection in public farm, Applied Mathematics and Computation, 237: 582-594, 2014.

[35] Pauline van den Driessche. Reproduction numbers of infectious disease models, Infectious Disease Modelling, 2(3): 288-303, 2017.

[36] C Connell McCluskey. Lyapunov functions for tuberculosis models with fast and slow progression, Mathematical Biosciences and Engineering, 3 : 1-12, 2006. 
[37] Medscape. Brucellosis Pathogenicity, https://emedicine.medscape.com/article/213430-overview, Accessed: 2018-11-07.

[38] Mariana N Xavier, Tatiane A Paixao, Andreas B den Hartigh, Renee M Tsolis, and Renato L Santos. Pathogenesis of Brucella spp., The open veterinary science journal, 4(1): 109-118, 2010.

[39] B Nannyonga, GG Mwanga, and LS Luboobi. An optimal control problem for ovine brucellosis with culling, Journal of biological dynamics, 9(1):198-214, 2015.

[40] Paride O Lolika, Chairat Modnak, and Steady Mushayabasa. On the dynamics of brucellosis infection in bison population with vertical transmission and culling, Mathematical biosciences,305: 42-54, 2018.

[41] Daniel Okuonghae, and Andrei Korobeinikov. Dynamics of tuberculosis: the effect of direct observation therapy strategy (DOTS) in Nigeria, Mathematical modelling of natural phenomena, 2(1): 113-128, 2007.

[42] P Padmanabhan, P Seshaiyer, and C Castillo-Chavez. Mathematical modeling, analysis and simulation of the spread of Zika with influence of sexual transmission and preventive measures, Letters in Biomathematics, 4(1): 148-166, 2017.

[43] Georgios Pappas, Photini Papadimitriou, Nikolaos Akritidis, Leonidas Christou, and Epameinondas V Tsianos. The new global map of human brucellosis, The Lancet infectious diseases, 6(2): 91-99, 2006.

[44] Felix Roth, Jakob Zinsstag, Dontor Orkhon, G Chimed-Ochir, Guy Hutton, Ottorino Cosivi, Guy Carrin, and Joachim Otte. Human health benefits from livestock vaccination for brucellosis: case study, Bulletin of the World health Organization, 81:867-876, 2003.

[45] E Schelling, C Diguimbaye, S Daoud, J Nicolet, P Boerlin, M Tanner, and J Zinsstag. Brucellosis and Q-fever seroprevalences of nomadic pastoralists and their livestock in Chad, Preventive veterinary medicine, 61(4): 279-293, 2003.

[46] Gabriel Mkilema Shirima. The epidemiology of brucellosis in animals and humans in Arusha and Manyara regions in Tanzania , PhD thesis. University of Glasgow, 2005.

[47] Gabriel M Shirima, Seleman N Masola, Obeid N Malangu, and Brant A Schumaker. Outbreak investigation and control case report of brucellosis: experience from livestock research centre, Mpwapwa, Tanzania, Onderstepoort Journal of Veterinary Research, 81(1):1-4, 2014

[48] J Kitaly. Bovine brucellosis in Government parastatal and Ujamaa village dairy farms in Central Zone of Tanzania: Assessment of Control measures in some farms In, Proceedings of the 2 nd Tanzania Veterinary Association Scientific Conference, 24-30, 1984.

[49] Emmanuel S Swai, and Luuk Schoonman. Human brucellosis: seroprevalence and risk factors related to high risk occupational groups in Tanga Municipality, Tanzania, Zoonoses and Public Health, 56(4):183-187, 2009.

[50] Gabriel Tumwine, Enock Matovu, John David Kabasa, David Okello Owiny, and Samuel Majalija. Human brucellosis: seroprevalence and associated risk factors in agro-pastoral communities of Kiboga District, Central Uganda, BMC public health, 15(1): 1-8, 2015.

[51] Pauline Van den Driessche, and James Watmough. Reproduction numbers and sub-threshold endemic equilibria for compartmental models of disease transmission, Mathematical biosciences, 180(1-2): 29-48, 2002.

[52] WHO. Brucellosis in humans and animals, http://http://www.who.int/csr/resources/publications/Brucellosis.pdf. Accessed: 2018-11-08.

[53] Melese Yilma, and Gezahegne Mamo, and Bedaso Mammo. Review on Brucellosis Sero-prevalence and Ecology in Livestock and Human Population of Ethiopia, Achievements in the Life Sciences, 10(1): 80-86, 2016.

[54] Fengbo Zhang, Zhiwei Li, Xiaolin La, Xiumin Ma, Yaoxin Zhang, Ping Ji, Min Jiang, Jinwei Hu, Zhaoxia Zhang, Xiaobo Lu, and Jianbing Ding. Multiple-locus variable-number tandem-repeat analysis of Brucella isolates from patients in Xinjiang China, International journal of clinical and experimental medicine, 8(9): 15716-15723, 2015.

[55] Juan Zhang, Gui-Quan Sun, Xiang-Dong Sun, Qiang Hou, Mingtao Li, Baoxu Huang, Haiyan Wang, and Zhen Jin. Prediction and control of brucellosis transmission of dairy cattle in Zhejiang Province, China, Plos one, 9(11): 1-13, 2014.

[56] J Zinsstag, and F Roth, D Orkhon, G Chimed-Ochir, M Nansalmaa, J Kolar, and P Vounatsou. A model of animal-human brucellosis transmission in Mongolia, Preventive veterinary medicine, 69(1-2): 77-95, 2005.

[57] FP Poester, LE Samartino, RL Santos. Pathogenesis and pathobiology of brucellosis in livestock, Rev Sci Tech, 32(1): 105-15, 2013.

[58] Wendi Wang, and Xiao-Qiang Zhao. Threshold dynamics for compartmental epidemic models in periodic environments, Journal of Dynamics and Differential Equations, 20(3): 699-717, 2008. 